\title{
Atypical Postpartum Stroke Presenting as Opalski Syndrome: Case Report and Review of the Literature
}

\author{
Mikael Levy Esther Levy Shimon Maimon \\ Interventional Neuroradiology Unit, Department of Neurosurgery, Tel Aviv \\ Sourasky Medical Center, Sackler Faculty of Medicine, Tel Aviv University, \\ Tel Aviv, Israel
}

\section{Key Words}

Post-delivery stroke $\cdot$ Opalski syndrome $\cdot$ Lateral medullary syndrome $\cdot$ Central sinus vein thrombosis · Pregnancy · Arterial dissection

\begin{abstract}
Background and Purpose: We present the first case of combined arterial (vertebral artery dissection) and venous [central sinus vein thrombosis (CSVT)] diseases presenting as Opalski syndrome in a female patient following induced delivery.

Case Description: A 32-year-old woman was admitted to our institute two weeks after induced delivery with intriguing neurological findings that were finally diagnosed as a combined venous-arterial disease. Although she was referred diagnosed with CSVT, her neurological findings indicated Wallenberg 'plus' syndrome with ipsilateral hemiparesis (Opalski syndrome), further confirmed by neuroimaging revealing arterial disease (vertebral artery dissection) combined with incidental acute CSVT. Coagulation, gynecological and cardiac problems were ruled out. Treatment consisted of continuous heparin with rigorous control of her blood pressure. Nine days later, the patient was discharged with prominent improvements. Most of the symptoms resolved following 3 months of rehabilitation.

Conclusions: Atypical strokes (such as Opalski syndrome) might present in postpartum patients. This rare diagnosis should be suspected in patients with Wallenberg 'plus' syndrome, and neuroimaging studies for determining the presence of arterial disease and brain stem lesions should be performed. Concomitant CSVT is rare and might mislead. Fine diagnosis followed by immediate conservative treatment can be of great benefit.
\end{abstract}




\section{Introduction}

Pregnant women might be in risk for central sinus vein thrombosis (CSVT) and vertebral artery dissection (VAD). Opalski syndrome, a rare variant of lateral medullary syndrome (LMS) with concomitant ipsilateral hemiparesis, results from the ipsilateral extension of the medullary infarct to the superior cervical cord caudal to the pyramidal decussation. Its etiology might be iatrogenic following induced delivery and coughing episodes [1]. A concomitant venous-arterial disease (Opalski syndrome and incidental CSVT) following induced delivery has not been reported previously. In our case, intriguing neurological findings followed by neuroimaging confirmed the diagnosis, leading to successful conservative treatment.

\section{Case Report}

A 32-year-old healthy woman with 2 previous normal deliveries and a recent induced vaginal delivery was referred from another hospital diagnosed with CSVT. Family and personal history was noncontributory, except for migraine, no smoking history and psychotherapy for anxiety. The latest pregnancy was normal up to the 28th week during which she complained of dyspnea and coughing (on supine position) that spontaneously resolved. No further complaints appeared until the 30th week when she suspected reduced fetal movement which was diagnosed as a nonviable fetus, leading to induced vaginal delivery on the following day. She was discharged 2 days later with no other complaints and no additional treatment.

One week later, headaches and neck tenderness (right side) appeared, for which conservative management was recommended. Four days later, she experienced dizziness, finger numbness and intermittent suffocation. Her unremarkable neurological examination in the emergency room (ER) led the physician to discharge her for ambulatory follow-up, diagnosed as 'mixed anxiety and depressive' reaction. Two days later, now complaining of blurred vision, vomiting and urinary retention, the patient was referred to the ER again and was admitted to the gynecology ward.

Her gynecological and ophthalmological evaluations were normal; $500 \mathrm{~cm}^{3}$ of urine was found in a post-voiding ultrasound, and an unexplained hypertension was treated. One day after admission, a new right hemiparesis (arm > leg) appeared. A CT venography (CTV) demonstrated CSVT of the left transverse and sigmoid sinuses (fig. 1il), with no signs of edema or hemorrhage. Heparin was initiated, and the patient was referred to our ER for 'evaluating the option of an endovascular treatment'.

We found discrepancies between the neurological signs and the isolated diagnosis of CSVT, and suspected brain stem involvement. A reevaluation of the CTV (that unintentionally included an arterial phase) revealed right VAD (V3-4 segments; fig. 1b), further confirmed by MRI showing an ischemic infarct damage of the brain stem (lateral and anterior medullary infarcts) and cerebellum, explaining the patient's symptoms (fig. 2 il-d). Concomitant incidental CSVT was confirmed. Aneurysms or stenosis were excluded.

On physical examination, tachycardia (heart rate 93) and hypertension (166/113 mm Hg) were noted. The patient was alert, suffering from headache and presenting the following neurological findings suggestive of Opalski syndrome: ipsilateral hemiparesis; vestibular signs (vertigo, diplopia, nystagmus and vomiting); cerebellar signs (side ataxia, dysdiadochokinesia, nystagmus and dysarthria); lateral spinothalamic tract signs (contralateral deficits in pain and temperature sensation, with preserved proprioception); nucleus ambiguus signs (dysphagia, hoarseness, palate and tongue deviation to the right); spinal trigeminal nucleus signs (ipsilateral loss of pain and temperature sensation from V2-3 distribution, with normal V1 and reduced sensation of the palate); bilateral positive Babinski signs; descending sympathetic fiber signs (partial ipsilateral Horner's syndrome: myosis and ptosis), and diplopia due to limited upward and lateral gaze of the right eye.

Heparin treatment maintained a PTT range of 60-70 s, followed by unfractionated heparin treatment ( $80 \mathrm{mg}$ twice a day) with rigorous blood pressure (BP) control (systolic 110-140 mm Hg). Gradual improvement could be noted, and the patient started to walk by day 4 and swallow by day 6 . 
A thorough workup included an echocardiography (mild tricuspid regurgitation), bilateral lower limb Doppler examination, hematological workup (CRP, folic acid, vitamin B12, homocysteine, factor V Leiden, cytoplasmic and perinuclear ANCA (antineutrophil cytoplasmic antibodies), rheumatoid factor, prothrombin, complement factors, anti-thrombin 3, $\beta$-2 glycoprotein, cardiolipin IgM and IgG, proteins $\mathrm{S}$ and $\mathrm{C}$, and lupus anticoagulant). All results were normal.

The patient was discharged after 9 days, further improving, being fully conscious and less hoarse, eating by herself, having less diplopia, nystagmus and hypoesthesia, and walking independently. After 3 months of rehabilitation, she resumed a normal lifestyle with further improvement on a recent MRI.

\section{Discussion}

\section{Opalski Syndrome}

Opalski [2] first described this phenomenon in 1946 as a variant of LMS with concomitant ipsilateral hemiparesis explained by the ipsilateral extension of the medullary infarct to the superior cervical cord caudal to the pyramidal decussation. Others have explained these combined findings as a result of vertebral artery (VA) occlusion/stenosis or VAD $[3,4]$, compromising the medullary penetrating arteries that arise from distal VAs or anterior spinal arteries [5]. Opalski syndrome might be due to either ischemic disturbances (such as distal VAD) and/or due to hemodynamically vulnerable areas such as the 'borderline zone' between the anterior and posterior spinal arteries and between the vertebral and spinal arteries [4].

Concomitant LMS with ipsilateral hemiparesis, supported by neuroimaging, led us to this rare diagnosis. Either the induced delivery and/or the unexplained coughs could have caused the VAD leading to brain stem lesions.

\section{CSVT, Stroke and Arterial Dissection during Puerperium}

Our patient presented a rare combination of VAD causing Opalski syndrome with incidental CSVT. Cases of dissections with or without concomitant CSVT following deliveries have been reported [6-12, 13-17]; however, our case differs from those found in the literature in that: (1) the gynecological course was more complicated [intrauterine fetal death (IUFD) and induced delivery]; (2) our patient experienced unexplained dyspnea and coughing 2 weeks before IUFD diagnosis; (3) our patient suffered from concomitant VAD, Opalski syndrome and CSVT, and (4) there were suspected findings of bilateral damages in the brain stem-spinal cord junction.

Women of child-bearing age have a higher incidence of stroke compared to agematched men [6, 10-12], with only mild differences between pregnant and nonpregnant patients [18]. Nonpregnant women have a lower incidence of hemorrhagic than ischemic strokes; however, these frequencies become similar during pregnancy, suggesting pregnancy as a risk factor for hemorrhagic strokes $[11,12]$. Strokes usually occur during the third trimester and the puerperium [18], up to 1 month postpartum [19]. Eclampsia/preeclampsia remains the main cause for both types of strokes (up to 47\%). Hemorrhagic strokes carry a poor prognosis [20,21]. 
During the peripartum period, the overall risk for strokes $(13.1-10.3 / 100,000)$ and CSVT $(11.6 / 100,000)$ is similar. Among nonpregnant aged-matched females, CSVT occur less frequently $[6,18]$, suggesting that in the peripartum period, women are prone to develop CSVT (approx. 5-20\% of all CSVT occur during this period [11]), mainly during the first 14 days postpartum [13]. Notably, CSVT by itself is considered as a cause for stroke in up to $38 \%$ of cases during the postpartum period $[6,9,14-16]$.

Another cause for ischemic strokes in young females is arterial dissection during pregnancy [18]. These might be associated with connective tissue disorders, trauma and aneurysms. Extracranial dissections commonly present as neck pain and headache (occipital and posterior cervical regions), and might be proceeded by ischemic symptoms within the first weeks of the dissection [22]. This gradual evolution can delay diagnosis.

Unlike carotid dissections, VAD more frequently extend intracranially and involve the subarachnoid space causing meningism. Less than $30 \%$ of the cases have simultaneous dissections of two or more vessels, for which an underlying connective tissue disorder should be suspected $[17,18]$.

Cervicocephalic arterial dissection (CCAD) is a rare postpartum complication, with only 16 reported cases to date [7-11,13-17]. The rate of spontaneous CCAD in the general population is $2.6 / 100,000$, of which $2.4 \%$ can occur during the postpartum period, meaning that there are $\sim 6.2 / 10,000,000$ annually $[9,23,24]$, representing $\sim 6 \%$ of spontaneous CCAD in women under 50 years of age. Extracranial dissections seem to occur more frequently among pregnant women [12]. Up to $90 \%$ of VAD might be complicated by embolic stroke [1].

Reversible cerebral vasoconstriction syndrome was associated with stroke during the postpartum period among 4/6 patients compared with only 3/96 aged-matched controls. Contrary to reversible cerebral vasoconstriction syndrome which has a favorable outcome, intracranial dissections in postpartum patients are characterized by a worse outcome [7].

The different occurrence between intra- and extradural dissections could possibly be explained by histological changes that appear once arteries (VAs and internal carotid arteries) become 'intradural': 'The change is characterized first by a diminution in thickness of the adventitial and medial coats, and second, by a very gross reduction or loss of elastic fibers in the media and external elastic lamina' [25].

There are two issues regarding the therapeutic course. The first issue concerns BP control. Our patient needed a tradeoff between BP reduction (due to the possible hemorrhagic transformation in the stroke area) and the general permissive hypertension (for dissections). The second issue concerns the usage of antiplatelet/anticoagulation drugs, to which caution was given due to the possible hemorrhagic transformation of a stroke. Once anticoagulation treatment is initiated, it will be generally replaced by antiplatelet therapy for approximately 6 months, due to the fact that most CCAD resolve after 3-6 months [26]. Neither therapy (antiplatelet vs. anticoagulant) was found superior for treating acute dissection $[26,27]$. Once the possibility of a connective tissue disorder is excluded, the risk of recurrence is $1 \%$ within $1.4-8.6$ years and up to $2 \%$ within the first months following the dissection [28-32]. 


\section{Induced Delivery Complications and Concerns about Coagulopathies}

Our patient presented vascular pathologies of two different origin: one of venous origin (CSVT) and one of arterial origin (VAD). Both could have been complications of the peripartum period and of the induced delivery. Hypercoagulability should also be considered since it can either cause or be a consequence of IUFD. Since no postmortem examination of the fetus was done, these issues as well as the reason for the IUFD remained unsolved.

Despite two previous normal pregnancies/deliveries and noncontributory personal/ familial coagulopathies, transient hemodynamic as well as hematologic changes during pregnancy and puerperium might be considered as additional causes. Pregnancy might alter all three factors of Virchow's triad (blood flow, blood vessel wall and constitution of blood) [33], and patients are therefore prone to develop vascular diseases, as seen in our patient.

\section{Anatomical Considerations (Posterior Circulation)}

Opalski syndrome is caused by compromised blood supply and/or hemodynamic vulnerability, both likely occurring during puerperium and/or induced delivery. It is well accepted that nearly all posterior inferior cerebellar artery (PICA) occlusions and more than $50 \%$ of VA occlusions (mainly if a PICA origin is included) might manifest as LMS. Importantly, $75 \%$ of all LMS cases are related to VA occlusion and $12 \%$ to pure PICA occlusions [22].

The last peridural VA segment is V3. The intradural V4 segment ascends to the lower pontine border and forms the basilar artery, giving rise to the PICA en route $(10 \mathrm{~mm}$ distal to the dura perforation). Our patient's right V3-4 dissection included the PICA, presenting as right medullary and cerebellar infarcts. This dissection might explain both constituents of Opalski syndrome: (1) LMS signs might be caused by the PICA-VArelated brain stem strokes, while (2) concomitant ipsilateral hemiparesis might be caused by the involvement of the anterior spinal artery arising from V4 that normally supplies the ventral two thirds of the spinal cord, including the pyramidal tract.

\section{Conclusion}

Combined venous-arterial disease should be considered among patients at high-risk for both pathologies, such as women during puerperium, even when their medical history is unremarkable. Discrepancies between neurological findings and incidental neuroimaging findings can mislead.

A clear-cut mechanism for the combined pathology could not be found; however, the combination of induced delivery combined with hypercoagulability and/or hemodynamic vulnerability during puerperium seems a reasonable option. It seems plausible that either the coughs and/or the induced delivery have caused the VAD that, in turn, led to Opalski syndrome. Pregnancy-related hypercoagulability might have been the reason for the concomitant CSVT. 
Conservative treatment combining BP control and anticoagulation seems an appropriate first step for these kinds of combined pathologies.
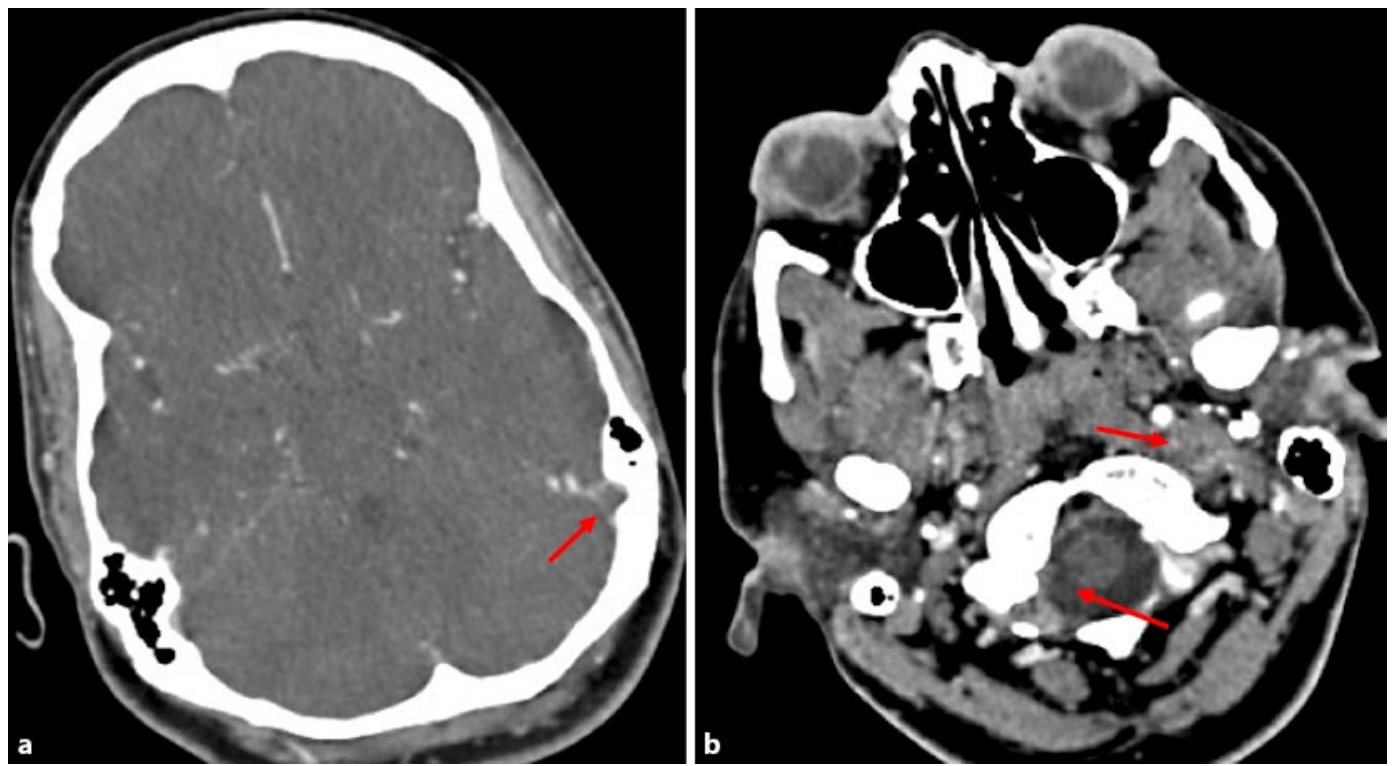

Fig. 1. a CTV showing left vein thrombosis. b CTV showing right VAD (V4 segment) and left jugular thrombosis. 

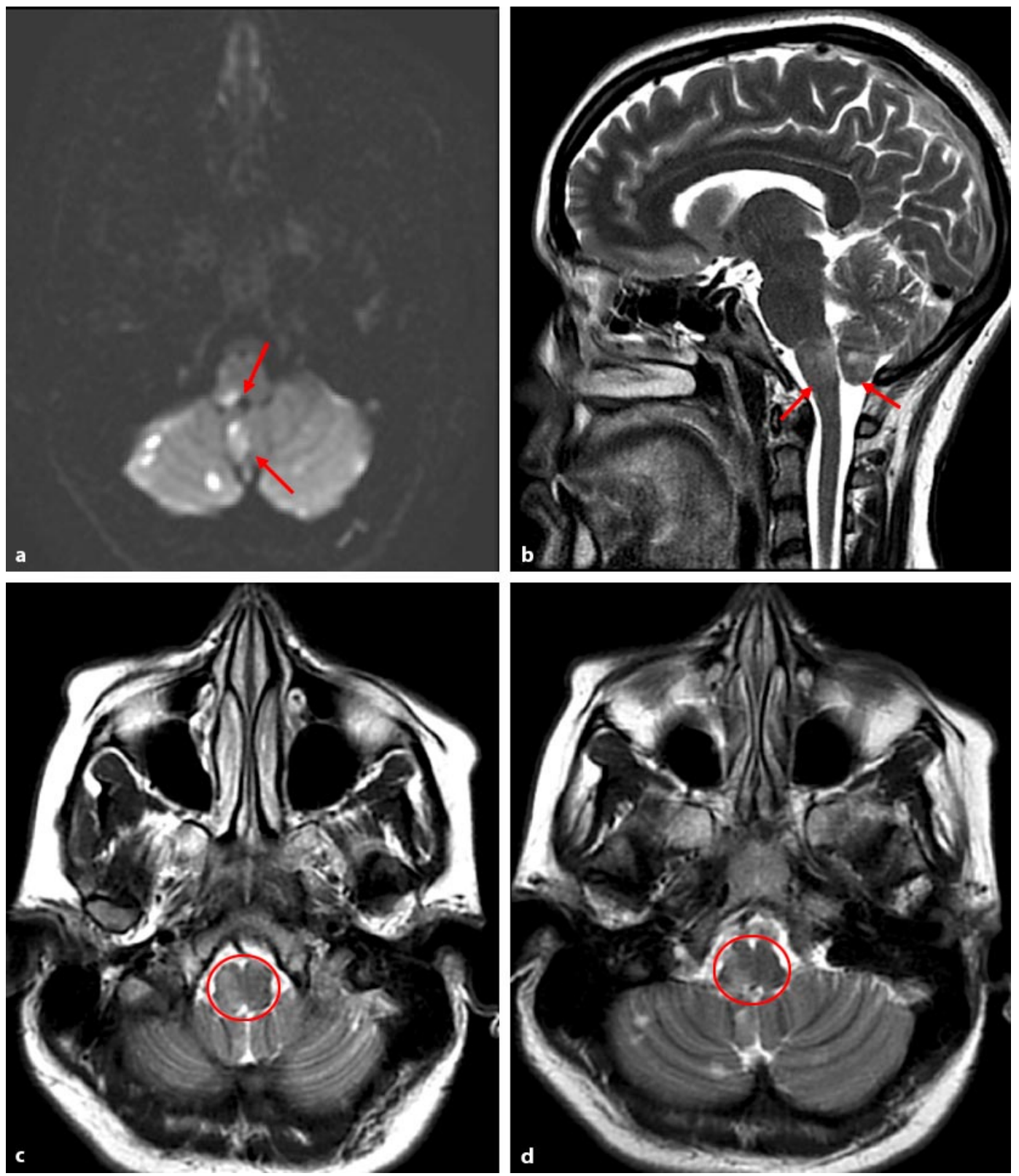

Fig. 2. a MRI (diffusion-weighted image) showing infarcts at the pyramidal decussating level. b MRI ( $\mathrm{T}_{2}$-weighted image) showing acute medullary and cerebellar infarcts. c MRI ( $\mathrm{T}_{2}$-weighted image) showing suspected bilateral infarcts at the pyramidal decussating level. d $\mathrm{MRI}$ ( $\mathrm{T}_{2}$-weighted image) showing acute medullary and cerebellar infarcts and CSVT.

\section{References}

1 Schievink WI: Spontaneous dissection of the carotid and vertebral arteries. N Engl J Med 2001;344:898-906.

2 Opalski A: Un nouveau syndrome sous-bulbaire partiell'artère vertébro-spinal postérieure. Paris Med 1946;1:214-220.

3 Montaner J, Alavarez-Sabin J: Opalski’s syndrome. J Neurol Neurosurg Psychiatry 1999;67:688-689. 
4 Dhamoon SK, Iqbal J, Collins GH: Ipsilateral hemiplegia and the Wallenberg syndrome. Arch Neurol 1984;41:179-180.

-5 Liu CY, Chang FC, Hu HH, Hsu LC: Ipsilateral crural monoparesis in lateral medullary infarction due to vertebral artery dissection. Eur J Neurol 2006;13:e8-e9.

-6 Ostrovskiy D, Hacein-Bey L, Varelas PN, Heverly DN: Simultaneous postpartum cerebral venous thrombosis and cervico-cephalic arterial dissections. Cerebrovasc Dis 2003;16:301-303.

7 Arnold M, Camus-Jacqmin M, Stapf C, Ducros A, Viswanathan A, Berthet K, Bousser MG: Postpartum cervicocephalic artery dissection. Stroke 2008;39:2377-2379.

8 McKinney JS, Messé SR, Pukenas BA, Satti SR, Weigele JB, Hurst RW, Levine JM, Kasner SE, Sansing LH: Intracranial vertebrobasilar artery dissection associated with postpartum angiopathy. Stroke Res Treat 2010;2010:320627.

- Jaigobin C, Silver FL: Stroke and pregnancy. Stroke 2000;31:2948-2951.

10 Gasecki AP, Kwiecinski H, Lyrer PA, Lynch TG, Baxter T: Dissections after childbirth. J Neurol 1999;246:712715.

-11 Sharshar T, Lamy C, Mas JL: Incidence and causes of strokes associated with pregnancy and puerperium. A study in public hospitals of Ile de France. Stroke 1995;26:930-936.

12 Mas JL, Bousser MG, Hasboun D, Laplane D: Extracranial vertebral artery dissections: a review of 13 cases. Stroke 1987;18:1037-1047.

13 Lanska DJ, Kryscio RJ: Risk factors for peripartum and postpartum stroke and intracranial venous thrombosis. Stroke 2000;31:1274-1282.

-14 Kittner SJ, Stern BJ, Feeser BR, Hebel R, Nagey DA, Buchholz DW, Earley CJ, Johnson CJ, Macko RF, Sloan MA, Witky RJ, Wozniak MA: Pregnancy and the risk of stroke. N Engl J Med 1996;335:768-774.

15 Wiebers DO, Mokri B: Internal carotid artery dissection after childbirth. Stroke 1985;16:956-959.

16 Van de Kelft E, Kunnen J, Truyen L, Heytens L: Postpartum dissecting aneurysm of the basilar artery. Stroke 1992;23:114-116.

17 Abisaab J, Nevadunsky N, Flomenbaum N: Emergency department presentation of bilateral carotid artery dissections in a postpartum patient. Ann Emerg Med 2004;44:484-489.

- 18 Awada A, Rajeh SA, Duarte R, Russell N: Stroke and pregnancy. Int J Gynecol Obstet 1995;48:157-161.

19 Lanska DJ, Kryscio RJ: Stroke and intracranial venous thrombosis during pregnancy and puerperium. Neurology 1998;51:1622-1628.

20 Witlin AG, Friedman SA, Egerman RS, Frangieh AY, Sibai BM: Cerebrovascular disorders complicating pregnancy - beyond eclampsia. Am J Obstet Gynecol 1997;176:1139-1148.

-21 Estanol B, Rodriguez A, Conte G, Aleman JM, Loyo M, Pizzuto J: Intracranial venous thrombosis in young women. Stroke 1979;10:680-684.

22 Fisher CM, Karnes WE, Kubik CS: Lateral medullary infarction: the pattern of vascular occlusion. J Neuropathol Exp Neurol 1961;20:323-379.

23 Schievink WI, Mokri B, Whisnant JP: Internal carotid artery dissection in a community: Rochester, Minnesota 1987-1992. Stroke 1993;24:1678-1680

24 Waidelich JM, Bullough AS, Mhyre JM: Internal carotid artery dissection: an unusual cause of postpartum headache. Int J Obstet Anesth 2008;17:61-65.

25 Wilkinson IM: The vertebral artery: extracranial and intracranial structure. Arch Neurol 1972;27:392-396.

26 Lyrer PA: Extracranial arterial dissection: anticoagulation is the treatment of choice: against. Stroke 2005;36:2042-2043.

27 Bogousslavsky J, Regli F: Ischemic stroke in adults younger than 30 years of age. Cause and prognosis. Arch Neurol 1987;44:479-482.

28 Perier O, Cauchie C, Demanet JC: Hématome intramural par dissection pariétale (aneursyme dissequant) du tronc basilaire. Acta Neurol Psychiatr Belg 1964;64:1064-1074.

29 Benninger DH, Georgiadis D, Kremer C, Studer A, Nedeltchev K, Baumgartner RW: Mechanism of ischemic infarct in spontaneous carotid dissection. Stroke 2004;35:482-485.

-30 Singhal AB: Postpartum angiopathy with reversible posterior leukoencephalopathy. Arch Neurol 2004;61:411416.

-31 Schievink WI, Bjornsson J, Piepgras DG: Coexistence of fibromuscular dysplasia and cystic medial necrosis in a patient with Marfan's syndrome and bilateral carotid artery dissections. Stroke 1994;25:2492-2496.

-32 Schievink WI, Mokri B, O'Fallon WM: Recurrent spontaneous cervical-artery dissection. N Engl J Med 1994;330:393-397.

33 Bourjeily G, Paidas M, Khalil H, Rosene-Montella K, Rodger M: Pulmonary embolism in pregnancy. Lancet 2010;375:500-512.

Mikael Levy and Shimon Maimon contributed equally to this work. 